\title{
Transcriptome sequencing and drought resistance gene annotation in Quercus liaotungensis leaves
}

\author{
Guobao Wang ${ }^{1} \cdot$ Li Qin $^{2}$
}

Received: 14 June 2020 / Revised: 17 February 2021 / Accepted: 16 July 2021 / Published online: 20 July 2021

(c) The Author(s) 2021

\begin{abstract}
Q. liaotungensis is an important drought-resistant tree species in Northeast China where the climate is dry and rainless. In this study, we performed a deep transcriptomic sequencing in $Q$. liaotungensis leaves, including de novo assembly and functional annotation for screening the candidate genes involved in drought avoidance. A total of 25,593 unigenes were obtained from Illumina sequencing platform. According to Gene Ontology annotation and KEGG pathway enrichment analysis, we screened a series of candidate genes encoding SOD, POD, CAT, DREB, MYB, WRKY, bZIP, and NAC from the $Q$. liaotungensis leaf transcriptome, all of which are potentially involved in drought resistance. The results of this study expanded the genetic resources of $Q$. liaotungensis and provided a theoretical basis for further exploring the functional gene information of $Q$. liaotungensis.
\end{abstract}

Keywords Quercus liaotungensis $\cdot$ Leaf · Transcriptome $\cdot$ Drought-related factors

\section{Introduction}

Oaks belong to the genus Quercus, which comprises several hundred diploid and highly heterozygous species spreading throughout the northern hemisphere, from the tropical to the boreal regions (Abrams 1990). Among the oak species, $Q$. liaotungensis, which leaf is the main food source for Antheraea pernyi, is an important drought-resistant tree species in the northern warm temperate zone. The leaf morphology of $Q$. liaotungensis is relative small which could adapt to the dry and rainless climate in Northeast China. However, a detailed characterization of genomic information of $Q$. liaotungensis leaf coping with drought is lacking.

Recent transcriptomic and gene expression profiling studies in oaks have led to the construction of large cDNA

Communicated by J. Zhao.

Li Qin

qinli1963@163.com

1 College of Biology and Agricultural Engineering, Weifang University, Weifang 261061, China, No. 5147 Dongfeng East Street

2 College of Bioscience and Biotechnology, Shenyang Agricultural University, No. 120 Dongling Road, Shenyang 110866, China libraries (Ueno et al. 2010; Kremer et al. 2012; Tarkka et al. 2013; Torre et al. 2014), and RNA-Seq studies have made it possible to identify genes involved in drought-resistant (Torre et al. 2014). In this study, the leaves of $Q$. liaotungensis were used for transcriptome analysis for expanding the genetic resources of $Q$. liaotungensis. Besides, a series of drought-related factors of $Q$. liaotungensis leaf were also screened. The results of this study may provide a theoretical basis for further research on drought resistance mechanism of $Q$. liaotungensis.

\section{Materials and methods}

\section{Sample preparation and RNA extraction}

Q. liaotungensis used in this study were located at the research base of Shenyang Agricultural University under natural environment at $23 \pm 2{ }^{\circ} \mathrm{C}$ with $70 \pm 5 \%$ relative humidity. Juvenile and mature leaves (Fig. 1A) from 30 individuals of 4-years old plants were collected and immediately frozen in liquid nitrogen and stored at $-80^{\circ} \mathrm{C}$ until processing. Total RNA was extracted using Trizol reagent (Invitrogen) according to the manual. The quantity and purity of RNA were analyzed using NanoDrop ND-1000 

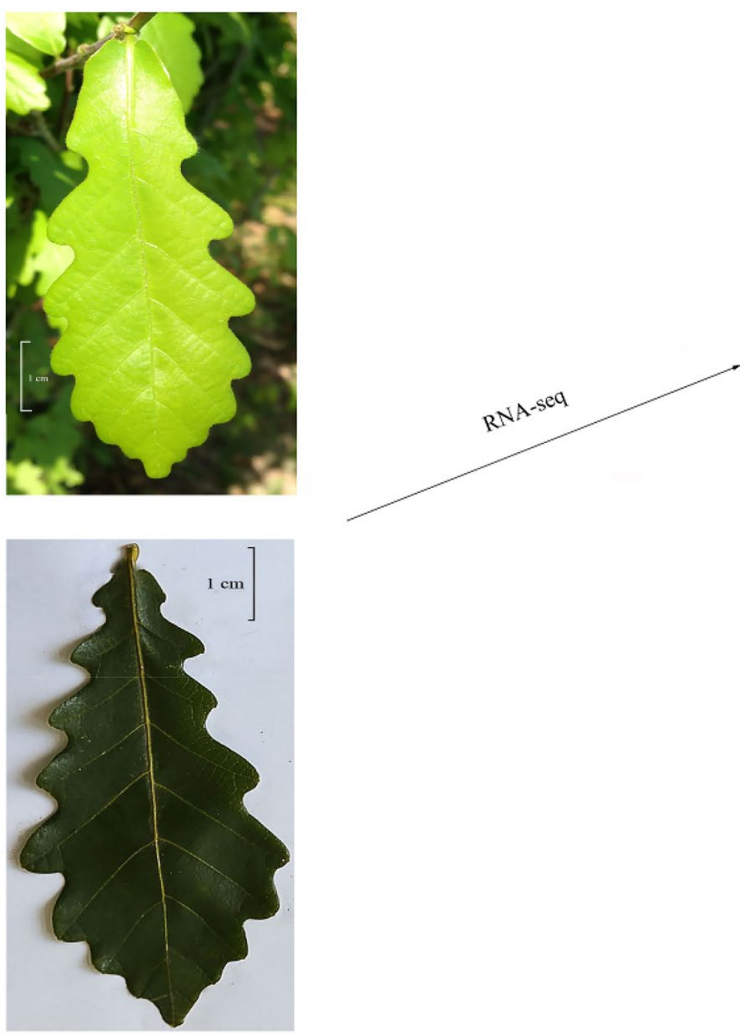

$\mathbf{A}$

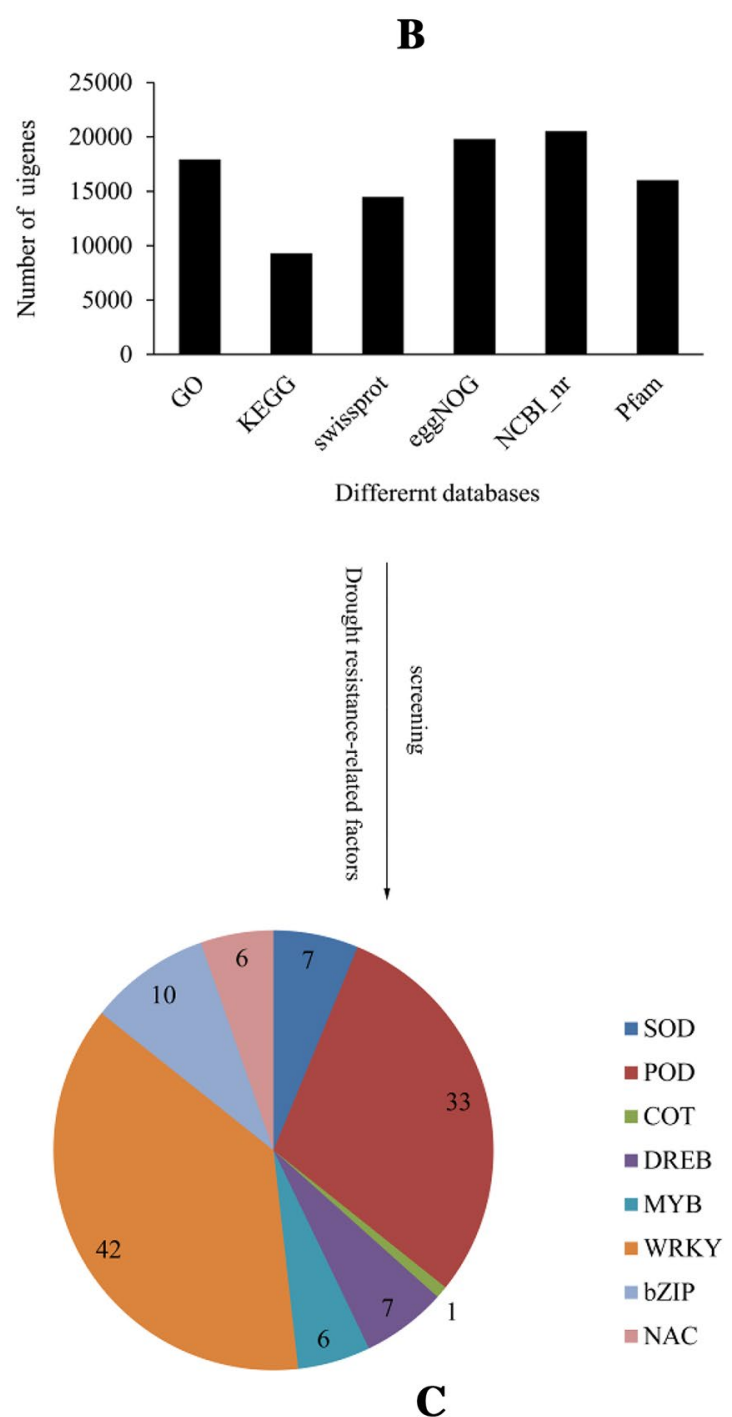

of different putative candidate factors involved in drought avoidance of $Q$. liaotungensis leaf

amount $>1 \mu \mathrm{g}$ was sent for cDNA library construction and RNA-seq which were commissioned by Lianchuan Biotechnology Co., Ltd (Hangzhou, China). Briefly, approximately $10 \mu \mathrm{g}$ of total RNA representing a specific adipose type was subjected to isolate Poly (A) mRNA with polyT oligo-attached magnetic beads (Invitrogen). Following purification, the poly(A)- or poly(A) + RNA fractions is fragmented into small pieces using divalent cations under 
elevated temperature. Then the cleaved RNA fragments were reverse-transcribed to create the final cDNA library in accordance with the protocol for the mRNA-seq sample preparation kit (Illumina, San Diego, USA), the average insert size for the paired-end libraries was $300 \mathrm{bp}$ $( \pm 50 \mathrm{bp})$. And then the paired-end sequencing was performed on an Illumina Hiseq 4000 (lc-bio, China).

\section{De novo transcriptome assembly}

The adaptor reads of the raw data were removed using Cutadapt software (https://cutadapt.readthedocs.io/en/stable/) (Martin 2011). The clean data were obtained by removing the low quality and repeat reads. The sequence quality was verified by FastQC software (http://www.bioinformatics.babraham.ac. uk/projects/FastQC/), including Q20, Q30, and GC content. The clean data were de novo assembled using Trinity software (Grabherr et al. 2011). Trinity grouped transcripts into clusters based on the shared sequence content. Each transcript cluster was referred to as a 'unigene', and the longest transcript sequence was chosen as the gene sequence of 'unigene'.

\section{Unigene annotation}

The annotations of the unigenes obtained from the assembled transcriptome were obtained by aligning against the databases including Gene ontology (http://www.geneontolo gy.org), Kyoto Encyclopedia of Genes and Genomes (http:// www.genome.jp/kegg/), eggnog (http://eggnogdb.embl.de/), NCBI_nr (http://www.ncbi.nlm.nih.gov/), and Pfam (http:// pfam.xfam.org/) using DIAMOND software (Buchfink et al. 2015).

\section{Validation of RNA-Seq by quantitative RT-PCR}

Ten unigenes were selected for qRT-PCR to validate the transcriptome data. Total RNA from the $Q$. liaotungensis leaf samples used for qRT-PCR were the same as for RNA-Seq. The gene-specific primers were designed using the predicted CDSs as reference sequences. qRT-PCR was performed on a CFX Connect ${ }^{\mathrm{TM}}$ Real-Time System (Bio-Rad) using a $20-\mu \mathrm{L}$ reaction system with a procedure as follows: $95^{\circ} \mathrm{C}$ for $30 \mathrm{~s}$, followed by 39 cycles of $95^{\circ} \mathrm{C}$ for $5 \mathrm{~s}, 60^{\circ} \mathrm{C}$ for $30 \mathrm{~s}$. Melting curves were generated after each run to confirm a single PCR product. Each reaction was run in triplicate. mRNA quantity of each gene was calculated with the $2^{-\Delta \mathrm{CT}}$ method (Livak and Schmittgen 2001).

\section{Results and discussion}

The RNA-seq data generated 54,153,182 raw reads. After filtering out the low-quality reads, 53,021,436 clean reads were obtained, which assembled into 41,207 transcripts with a mean length of $704 \mathrm{bp}$ and GC content of $42.17 \%$, and 25,593 unigenes with a mean length of $687 \mathrm{bp}$ and GC content of $42.31 \%$, based on Trinity assembly platform (Table 1). The raw sequence reads of this study were deposited in the Gene Expression Omnibus (GEO) under the accession number GSE125798. According to the Q. liaotungensis leaf transcriptome, 16,021, 17,935, 9290, 14,476, 19,806, 20,529 unigenes were matched to the Pfam, GO, KEGG, Swiss-Prot, eggNOG, NCBI_nr database, respectively (Table 2, Fig. 1B). The species distribution of the unigenes via blasting into NCBI_nr is shown in Fig. 2. The species that most contributed to the annotation was Juglans regia (59.43\%), following with Ziziphus jujuba (4.04\%), Vitis vinifera (3.52\%), Theobroma cacao (3.3\%), Prunus persica (2.41\%), Prunus mume (2.07\%), which suggested that there may be a closely genetic relationship between $Q$. liaotungensis and J. regia. The qRT-PCR results showed that the expression patterns of the candidate genes were consistent with those from RNA-Seq (Fig. 3),

Table 2 Unigenes annotated in different databases

\begin{tabular}{lcc}
\hline Database & Number of unigenes & Ratio $(\%)$ \\
\hline All & 25,593 & 100.00 \\
GO & 17,935 & 70.08 \\
KEGG & 9290 & 36.30 \\
swissprot & 14,476 & 56.56 \\
eggNOG & 19,806 & 77.39 \\
NCBI_nr & 20,529 & 80.21 \\
Pfam & 16,021 & 62.60 \\
\hline
\end{tabular}

Table 1 Statistical analysis of the transcriptome sequence data

\begin{tabular}{llllllll}
\hline Index & All & GC\% & Min length & Median length & Max length & Total assembled bases & N50 \\
\hline Transcript & 41,207 & 42.17 & 201 & 704 & 12,190 & $41,133,221$ & 1515 \\
Gene & 25,593 & 42.31 & 201 & 687 & 12,190 & $25,541,255$ & 1550 \\
\hline
\end{tabular}


Fig. 2 Species distribution of the unigenes via blasting into NCBI_nr database. Different colors represent different species. The number represents the proportion of unigene matched to different species

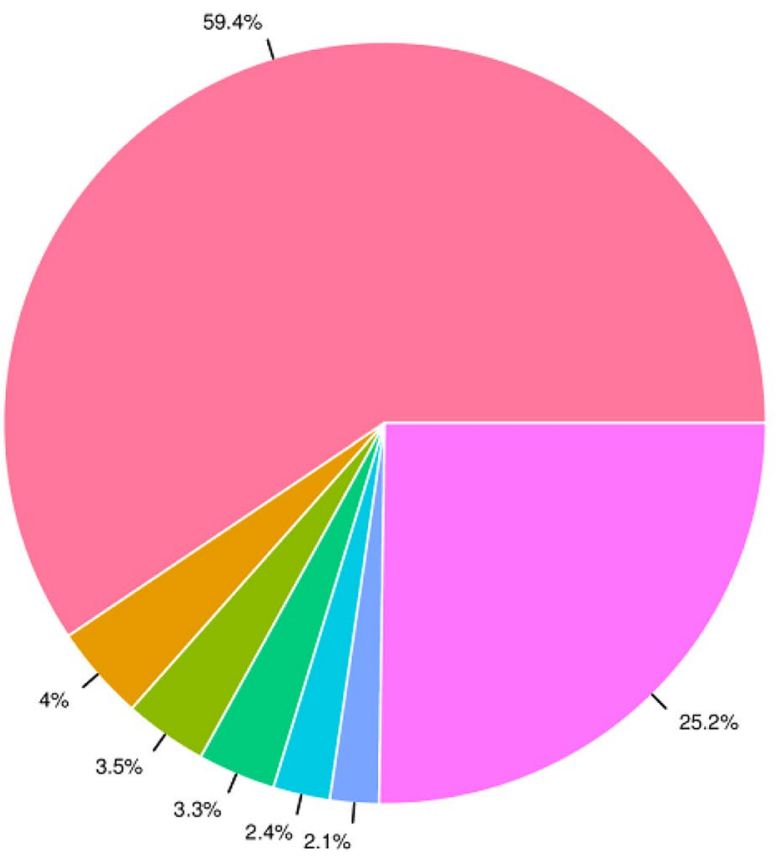

Juglans_regia $(59.43 \%)$

Ziziphus jujuba (4.04\%)

- Vitis_vinifera $(3.52 \%)$

- Theobroma cacao $(3.3 \%)$

- Prunus persica (2.41\%)

- Prunus mume $(2.07 \%)$

- Others $(25.23 \%)$

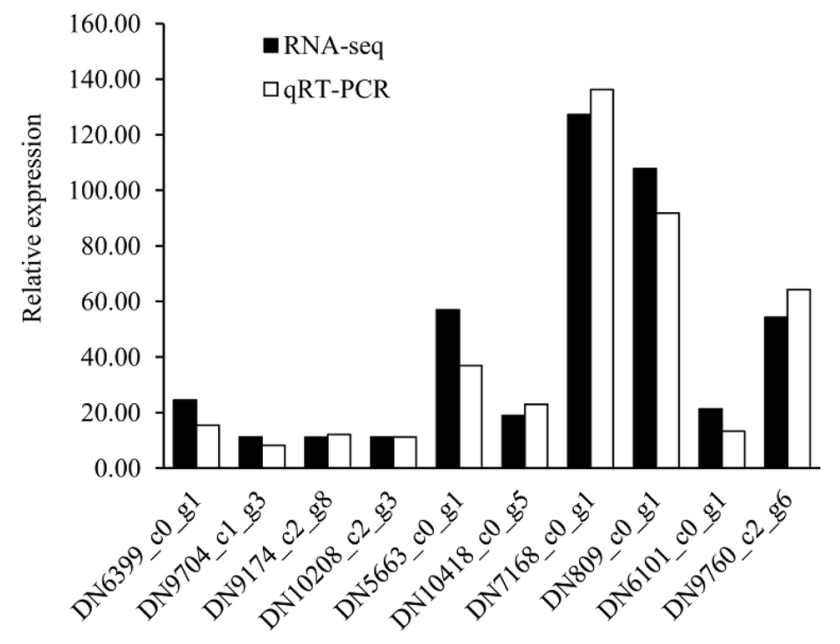

Fig. 3 Verification of the selected unigenes by qRT-PCR as compared with RNA-Seq data. $X$-axis represents the 10 genes selected for qRTPCR validation. $Y$-axis represents the relative expression. DN6399_ c0_g1, a superoxide dismutase 5 [Betula platyphylla]. DN9704 c1_g3, putative peroxidase 48 [Juglans regia]. DN9174_c2_g8, PREDICTED: basic leucine zipper 61 [Juglans regia]. DN10208_c2_ g3, PREDICTED: putative peroxidase 48 [Juglans regia]. DN5663_ c0_g1, PREDICTED: putative dehydration-responsive elementbinding protein $2 \mathrm{H}$ [Juglans regia]. DN10418_c0_g5, PREDICTED: probable WRKY transcription factor 23 [Juglans regia]. DN7168_ c0_g1, PREDICTED: probable WRKY transcription factor 75 [ Juglans regia]. DN809_c0_g1, PREDICTED: bZIP transcription factor 53 [Juglans regia]. DN6101_c0_g1, PREDICTED: basic leucine zipper 23 [Vitis vinifera]. DN9760_c2_g6, PREDICTED: transcription factor MYB44 [Juglans regia] confirming the expression of the unigenes identified in the deep sequencing analysis.

The unigenes were classified into three categories including cellular component, molecular function and biological process via GO analysis, with 675, 1905 and 2849 GO terms corresponding to each category, respectively. The top 25 significantly clustered GO terms of the unigenes for each category are shown in Fig. 4. Among the subcategories of cellular component, assignments were mostly given to nucleus, integral component of membrane, plasma membrane, cytoplasm, chloroplast, mitochondrion, and cytosol. The majority of the annotated unigenes were assigned to protein binding, ATP binding, protein serine/threonine kinase activity and DNA binding in molecular function. Dominant GO terms of the biological process subcategories were grouped into regulation of transcription, defense response, protein phosphorylation, oxidation-reduction process, and signal transduction.

KEGG pathway enrichment analysis was conducted for unigenes obtained from the transcriptome. The results showed that the unigenes in $Q$. liaotungensis could be assigned to 138 pathways. Among the 25 top KEGG pathways with the highest representation of unigenes, the abundant genes mapped onto endocytosis, plant hormone signal transduction, starch and sucrose metabolism, RNA transport, biosynthesis of amino acids, carbon metabolism, and amino sugar and nucleotide sugar metabolism (Fig. 5).

We screened a series of candidate genes potentially involved in drought adaptation via GO and KEGG analysis 

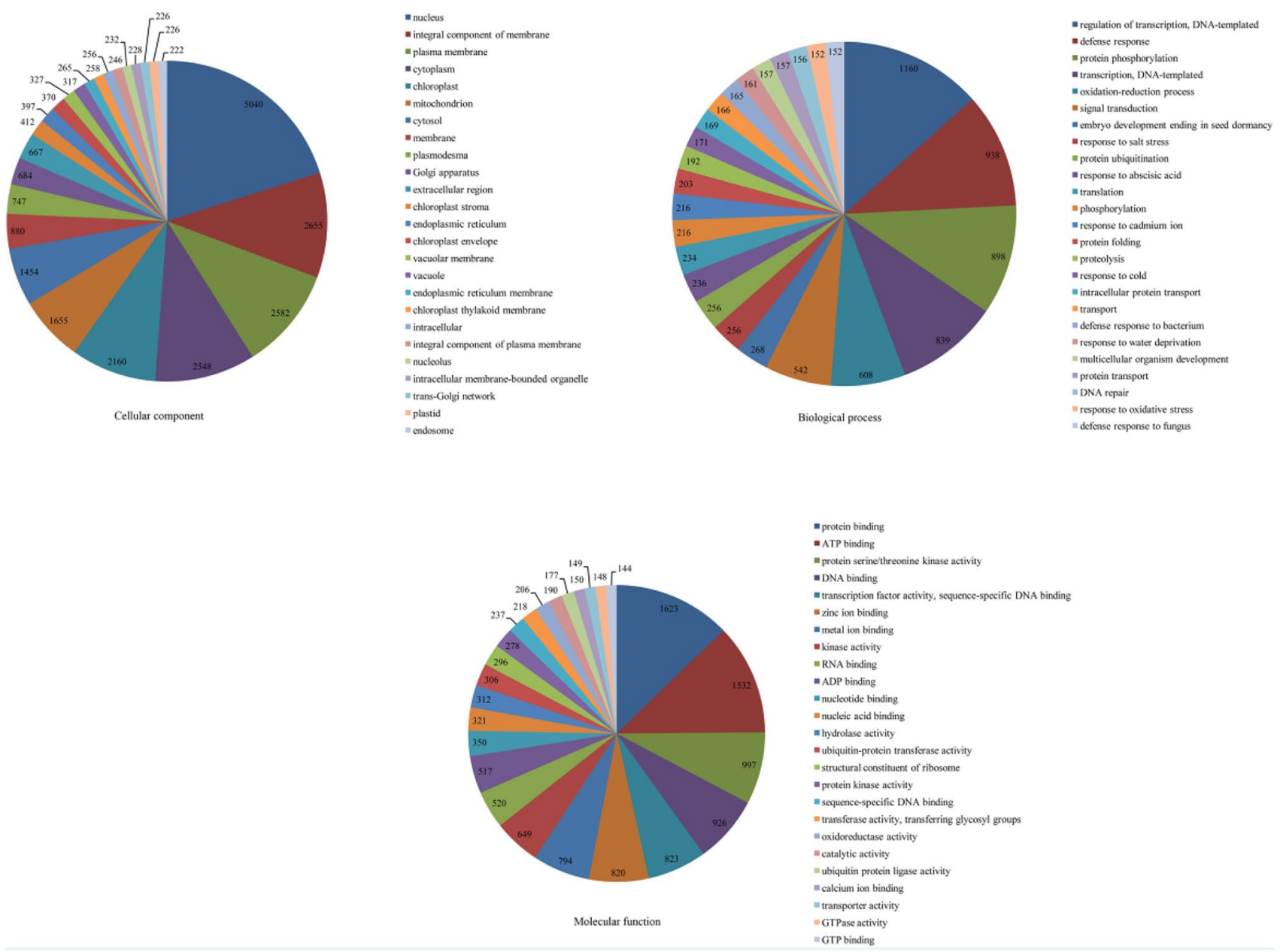

Fig. 4 The top 25 enriched GO terms in cellular component, molecular function and biological process categories of the unigenes from $Q$. liaotungensis leaf transcriptome

from those unigenes, including the genes encoding superoxide dismutase (SOD), peroxidase (POD), catalase (CAT), and several drought resistance-related transcription factors (TFs) such as dehydration-responsive element binding (DREB), v-myb avian myeloblastosis viral oncogene homolog (MYB), WRKY, basic leucine zipper (bZIP), and NAC (Fig. 1C, Table 3).

Drought has several effects on plant growth and development, one of which is oxidative damage. We identified 41 genes encoding superoxide dismutase (SOD), peroxidase (POD) and catalase (CAT) (Table 3) which compose the antioxidant defense system in plants that can cooperate to resist the damage of active oxygen to cells. Seven candidate 
Fig. 5 The top 25 enriched pathways of the unigenes from Q. liaotungensis leaf transcriptome

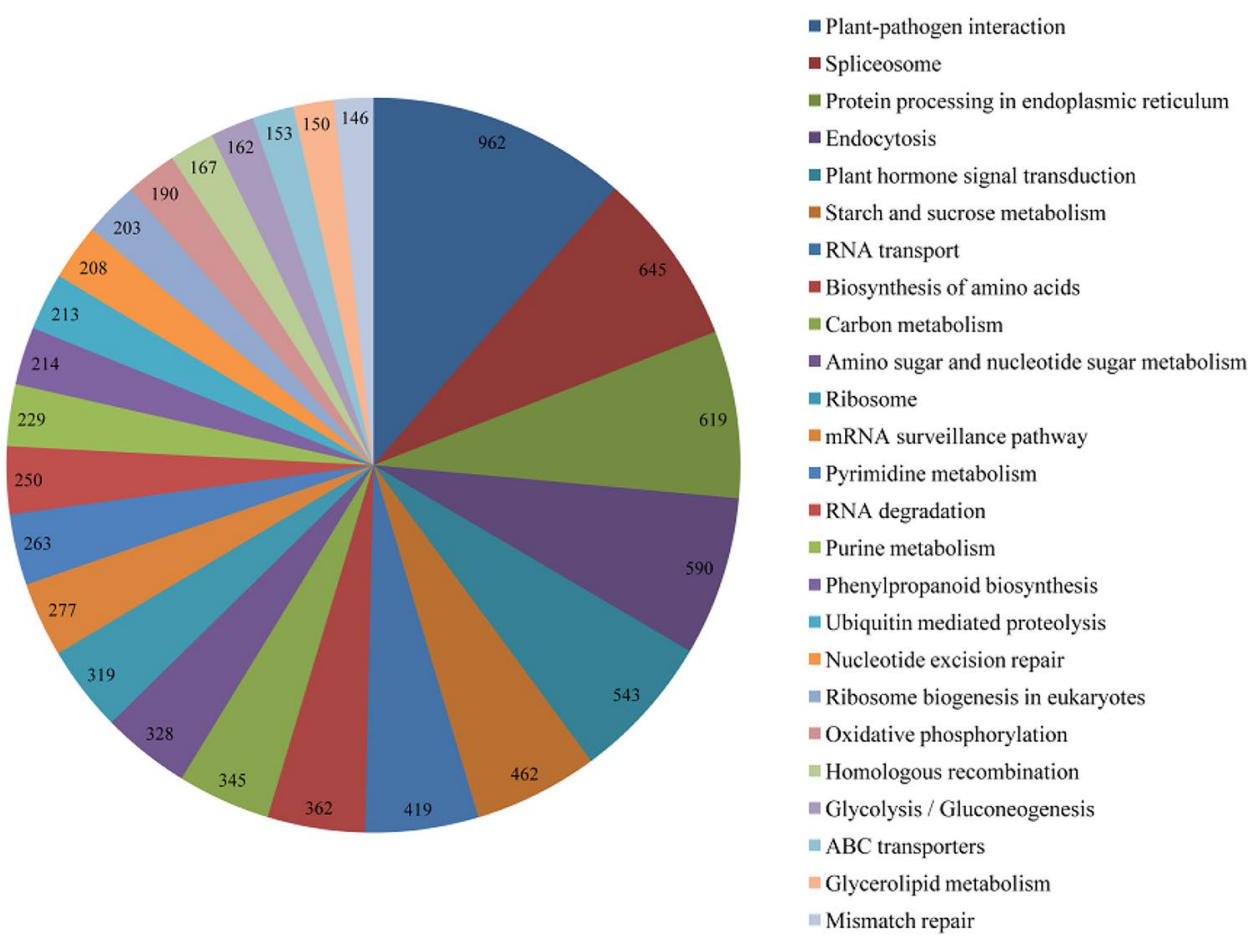

genes encoding DREBs (Table 3) including DREB1 and DREB2 were obtained. Overexpression of DREB1 increased tolerance of Malus baccata to low temperature, drought, and salt stresses (Yang et al. 2010). When treated with dehydration, the expression of DREB2A increased in Arabidopsis thaliana (Sakuma et al. 2007). Several drought resistancerelated TFs such as MYB and bZIP which were founded in Quercus pubescens leaves (Torre et al. 2014) were also identified in this study. We screened 5 genes encoding MYB TFs, which is one of the largest transcription factor families and play regulatory roles in developmental processes and defense responses in plants, including MYB108, 24, 44, 12 (Table 3). The bZIP and NAC TFs are known to play a crucial role in response to various processes in plant as well as abiotic or biotic stress challenges such as drought (Xiang et al. 2008; Huang et al. 2015). WRKY proteins are newly identified TFs that are also involved in drought tolerance in plants (Ren et al. 2010). Overall, we screened 10, 42, and 6 candidate genes encoding bZIPs, WRKYs, and NACs, respectively (Table 3 ).

Through high-throughput sequencing, we can quickly obtain the desired target genes. As a consequence, the results of this study expanded the genetic resources of $Q$. 
Table 3 The putative candidate genes involved in drought avoidance

\begin{tabular}{|c|c|}
\hline Gene ID & Annotation \\
\hline DN6399_c0_g1 & Superoxide dismutase 5 [Betula platyphylla] \\
\hline DN7263_c0_g1 & PREDICTED: superoxide dismutase [Fe], chloroplastic [Juglans regia] \\
\hline DN7744_c0_g1 & Superoxide dismutase, partial [Fagus sylvatica $]$ \\
\hline DN11311_c2_g3 & $\mathrm{Cu} / \mathrm{Zn}$-superoxide dismutase copper chaperone precursor [Corylus heterophylla] \\
\hline DN4365_c0_g1 & PREDICTED: superoxide dismutase [Cu-Zn] [Juglans regia] \\
\hline DN7248_c0_g1 & PREDICTED: superoxide dismutase [Cu-Zn], chloroplastic [Fragaria vesca subsp. vesca] \\
\hline DN10225_c2_g4 & $[\mathrm{Fe}]$ superoxide dismutase, partial [Betula platyphylla $]$ \\
\hline DN8775_c0_g1 & PREDICTED: L-ascorbate peroxidase 2, cytosolic-like [Juglans regia] \\
\hline DN3904_c0_g1 & PREDICTED: L-ascorbate peroxidase 3, peroxisomal-like [Juglans regia] \\
\hline DN9185_c0_g11 & PREDICTED: probable L-ascorbate peroxidase 6, chloroplastic isoform X2 [Ziziphus jujuba] \\
\hline DN10135_c1_g3 & PREDICTED: L-ascorbate peroxidase, cytosolic [Gossypium raimondii] \\
\hline DN7015_c0_g1 & PREDICTED: cationic peroxidase 1-like [Lupinus angustifolius] \\
\hline DN10800_c0_g1 & PREDICTED: probable glutathione peroxidase 2 [Juglans regia $]$ \\
\hline DN7056_c0_g1 & PREDICTED: phospholipid hydroperoxide glutathione peroxidase 1, chloroplastic-like [Juglans regia] \\
\hline DN10459_c2_g1 & PREDICTED: putative L-ascorbate peroxidase 6 [Juglans regia $]$ \\
\hline DN8556_c0_g1 & PREDICTED: peroxidase A2-like [Juglans regia] \\
\hline DN7254_c0_g1 & PREDICTED: peroxidase 25-like [Juglans regia] \\
\hline DN9704_c1_g3 & putative peroxidase 48 [Juglans regia] \\
\hline DN11127_c1_g2 & PREDICTED: peroxidase 73-like [Juglans regia] \\
\hline DN277_c0_g1 & PREDICTED: peroxidase 47 [Juglans regia] \\
\hline DN11207_c1_g2 & PREDICTED: putative peroxidase 48 [Juglans regia] \\
\hline DN9110_c0_g1 & PREDICTED: peroxidase 17-like [Juglans regia] \\
\hline DN10911_c0_g1 & PREDICTED: peroxidase 3-like [Juglans regia] \\
\hline DN11029_c1_g2 & PREDICTED: putative peroxidase 48 [Juglans regia] \\
\hline DN4001_c0_g1 & peroxidase [Betula platyphylla] \\
\hline DN11503_c2_g1 & PREDICTED: putative peroxidase 48 [Malus domestica] \\
\hline DN5675_c0_g1 & PREDICTED: peroxidase P7-like [Populus euphratica] \\
\hline DN2342_c0_g1 & PREDICTED: peroxidase 55 [Vitis vinifera] \\
\hline DN10208_c2_g5 & PREDICTED: putative peroxidase 48 [Ziziphus jujuba] \\
\hline DN7165_c0_g1 & PREDICTED: peroxidase 31-like [Juglans regia] \\
\hline DN7165_c0_g2 & PREDICTED: peroxidase 31-like [Juglans regia] \\
\hline DN9704_c1_g1 & PREDICTED: putative peroxidase 48 [Ziziphus jujuba] \\
\hline DN11057_c0_g2 & PREDICTED: peroxidase 4-like [Vitis vinifera] \\
\hline DN11457_c0_g2 & PREDICTED: putative peroxidase 48 [Glycine max] \\
\hline DN10208_c2_g3 & PREDICTED: putative peroxidase 48 [Juglans regia] \\
\hline DN11661_c0_g1 & PREDICTED: peroxidase 4-like [Juglans regia] \\
\hline DN8556_c0_g1 & PREDICTED: peroxidase A2-like [Juglans regia] \\
\hline DN11057_c0_g2 & PREDICTED: peroxidase 4-like [Vitis vinifera] \\
\hline DN9704_c1_g2 & PREDICTED: putative peroxidase 48 [Malus domestica] \\
\hline DN11029_c1_g3 & PREDICTED: putative peroxidase 48 [Juglans regia] \\
\hline DN10358_c0_g3 & PREDICTED: catalase isozyme 1 [Juglans regia] \\
\hline DN9997_c2_g1 & transcription factor MYB5 [Fragaria $x$ ananassa] \\
\hline DN10502_c1_g1 & PREDICTED: transcription factor MYB108-like [Populus euphratica] \\
\hline DN330_c0_g1 & PREDICTED: transcription factor MYB24-like [Juglans regia] \\
\hline DN9760_c2_g6 & PREDICTED: transcription factor MYB44 [Juglans regia] \\
\hline DN1817_c0_g1 & PREDICTED: transcription factor MYB108-like [Juglans regia] \\
\hline DN10064_c2_g2 & PREDICTED: transcription factor MYB12-like isoform X2 [Juglans regia] \\
\hline DN9464_c0_g1 & PREDICTED: dehydration-responsive element-binding protein 1A-like [Juglans regia] \\
\hline DN5663_c0_g1 & PREDICTED: putative dehydration-responsive element-binding protein $2 \mathrm{H}$ [Juglans regia] \\
\hline DN5351_c0_g1 & PREDICTED: dehydration-responsive element-binding protein 2A-like isoform X1 [Juglans regia] \\
\hline
\end{tabular}


Table 3 (continued)

\begin{tabular}{|c|c|}
\hline Gene ID & Annotation \\
\hline DN10470_c0_g2 & PREDICTED: dehydration-responsive element-binding protein 1D-like [Nelumbo nucifera] \\
\hline DN5663_c0_g3 & Dehydration-responsive element-binding protein 2A [Carica papaya $]$ \\
\hline DN10595_c0_g6 & PREDICTED: dehydration-responsive element-binding protein 1A-like [Ziziphus jujuba] \\
\hline DN10470_c0_g4 & PREDICTED: dehydration-responsive element-binding protein 1A-like [Nicotiana attenuata] \\
\hline DN9174_c2_g8 & PREDICTED: basic leucine zipper 61 [Juglans regia] \\
\hline DN1381_c0_g1 & PREDICTED: basic leucine zipper 25 [Juglans regia] \\
\hline DN11089_c0_g2 & PREDICTED: basic leucine zipper 23-like [Juglans regia] \\
\hline DN6101_c0_g1 & PREDICTED: basic leucine zipper 23 [Vitis vinifera] \\
\hline DN6968_c0_g1 & PREDICTED: bZIP transcription factor 53 [Ricinus communis] \\
\hline DN9193_c0_g1 & PREDICTED: bZIP transcription factor 17 [Juglans regia] \\
\hline DN9677_c0_g1 & PREDICTED: bZIP transcription factor 53-like [Prunus mume] \\
\hline DN10955_c1_g4 & PREDICTED: bZIP transcription factor 60-like [Juglans regia] \\
\hline DN8966_c0_g2 & PREDICTED: bZIP transcription factor 16-like [Juglans regia] \\
\hline DN809_c0_g1 & PREDICTED: bZIP transcription factor 53 [Juglans regia] \\
\hline DN11516_c0_g1 & PREDICTED: NAC transcription factor 29-like [Juglans regia] \\
\hline DN11389_c0_g2 & PREDICTED: NAC transcription factor 56 [Juglans regia] \\
\hline DN10815_c0_g1 & NAC transcription factor, partial [Fagus sylvatica] \\
\hline DN11389_c0_g4 & PREDICTED: NAC transcription factor 56 [Juglans regia] \\
\hline DN1786_c0_g1 & PREDICTED: NAC transcription factor 25 [Juglans regia] \\
\hline DN1874_c0_g1 & PREDICTED: NAC transcription factor 29 [Theobroma cacao] \\
\hline DN3070_c0_g1 & PREDICTED: probable WRKY transcription factor 29 isoform X2 [Juglans regia] \\
\hline DN10418_c0_g5 & PREDICTED: probable WRKY transcription factor 23 [Juglans regia] \\
\hline DN9002_c6_g3 & PREDICTED: probable WRKY transcription factor 33 [Juglans regia] \\
\hline DN10303_c3_g4 & PREDICTED: probable WRKY transcription factor 32 isoform X2 [Vitis vinifera] \\
\hline DN7541_c0_g2 & PREDICTED: probable WRKY transcription factor 2 [Prunus mume] \\
\hline DN4882_c0_g1 & PREDICTED: probable WRKY transcription factor 40 [Juglans regia] \\
\hline DN9140_c1_g1 & PREDICTED: probable WRKY transcription factor 53 [Juglans regia] \\
\hline DN6992_c0_g1 & PREDICTED: probable WRKY transcription factor 51 isoform X1 [Juglans regia] \\
\hline DN9650_c1_g6 & PREDICTED: probable WRKY transcription factor 21 [Juglans regia] \\
\hline DN9208_c4_g1 & PREDICTED: probable WRKY transcription factor 57 [Juglans regia] \\
\hline DN10157_c0_g3 & PREDICTED: probable WRKY transcription factor 3 isoform X1 [Juglans regia] \\
\hline DN8858_c1_g5 & PREDICTED: probable WRKY transcription factor 13 [Theobroma cacao] \\
\hline DN11151_c2_g4 & PREDICTED: probable WRKY transcription factor 65 [Juglans regia] \\
\hline DN9530_c2_g5 & PREDICTED: probable WRKY transcription factor 31 [Juglans regia] \\
\hline DN9986_c3_g1 & PREDICTED: probable WRKY transcription factor 31 [Nelumbo nucifera] \\
\hline DN9087_c2_g1 & PREDICTED: probable WRKY transcription factor 4 [Juglans regia] \\
\hline DN9333_c1_g1 & PREDICTED: probable WRKY transcription factor 7 [Juglans regia] \\
\hline DN9569_c1_g2 & WRKY transcription factor 43 [Manihot esculenta] \\
\hline DN9140_c2_g2 & PREDICTED: probable WRKY transcription factor 20 isoform X1 [Juglans regia] \\
\hline DN9632_c0_g4 & PREDICTED: probable WRKY transcription factor 4 [Juglans regia] \\
\hline DN9002_c4_g1 & PREDICTED: probable WRKY transcription factor 20 isoform X1 [Juglans regia] \\
\hline DN10418_c0_g4 & PREDICTED: probable WRKY transcription factor 48 [Juglans regia] \\
\hline DN9553_c1_g6 & PREDICTED: probable WRKY transcription factor 70 isoform X2 [Juglans regia] \\
\hline DN9282_c1_g2 & PREDICTED: WRKY transcription factor 44-like [Juglans regia] \\
\hline DN7168_c0_g1 & PREDICTED: probable WRKY transcription factor 75 [Juglans regia] \\
\hline DN9652_c4_g3 & PREDICTED: probable WRKY transcription factor 28 [Juglans regia] \\
\hline DN2165_c0_g1 & PREDICTED: probable WRKY transcription factor 69 [Ziziphus jujuba] \\
\hline DN9423_c0_g1 & PREDICTED: probable WRKY transcription factor 31 isoform X2 [Juglans regia] \\
\hline DN11844_c0_g1 & PREDICTED: probable WRKY transcription factor 61 [Juglans regia] \\
\hline DN8225_c0_g1 & PREDICTED: WRKY transcription factor 55-like [Juglans regia] \\
\hline
\end{tabular}


Table 3 (continued)

\begin{tabular}{ll}
\hline Gene ID & Annotation \\
\hline DN9947_c0_g1 & PREDICTED: probable WRKY transcription factor 50 isoform X2 [Prunus mume] \\
DN9148_c1_g5 & PREDICTED: probable WRKY transcription factor 2 [Juglans regia] \\
DN9553_c1_g9 & PREDICTED: probable WRKY transcription factor 70 [Juglans regia] \\
DN9279_c4_g6 & WRKY transcription factor 47-2 [Dimocarpus longan] \\
DN9002_c7_g3 & PREDICTED: WRKY transcription factor 1 isoform X1 [Juglans regia] \\
DN9767_c3_g7 & PREDICTED: probable WRKY transcription factor 53 [Juglans regia] \\
DN4756_c0_g1 & PREDICTED: probable WRKY transcription factor 69 isoform X1 [Juglans regia] \\
DN7477_c0_g1 & PREDICTED: WRKY transcription factor 44-like [Nelumbo nucifera] \\
DN10872_c0_g11 & PREDICTED: probable WRKY transcription factor 53 [Juglans regia] \\
DN9899_c0_g5 & PREDICTED: probable WRKY transcription factor 40 [Juglans regia] \\
DN9140_c2_g8 & PREDICTED: WRKY transcription factor WRKY24-like isoform X2 [Juglans regia] \\
DN10364_c0_g1 & PREDICTED: WRKY transcription factor 18-like [Juglans regia] \\
\hline
\end{tabular}

liaotungensis and provided useful information for further research on drought resistance mechanisms of $Q$. liaotungensis.

Author contribution statement GW and LQ conceived the project; GW performed the experiments; GW analyzed the data and wrote the paper.

Acknowledgements This work was supported by the Natural Science Foundation of Shandong Province (ZR2020QC190), Weifang University Doctor Startup Fund (2020BS26) and the National Modern Agriculture Industry Technology System Construction Project (Silkworm and Mulberry) (CARS-18).

Open Access This article is licensed under a Creative Commons Attribution 4.0 International License, which permits use, sharing, adaptation, distribution and reproduction in any medium or format, as long as you give appropriate credit to the original author(s) and the source, provide a link to the Creative Commons licence, and indicate if changes were made. The images or other third party material in this article are included in the article's Creative Commons licence, unless indicated otherwise in a credit line to the material. If material is not included in the article's Creative Commons licence and your intended use is not permitted by statutory regulation or exceeds the permitted use, you will need to obtain permission directly from the copyright holder. To view a copy of this licence, visit http://creativecommons.org/licenses/by/4.0/.

\section{References}

Abrams MD (1990) Adaptations and responses to drought in Quercus species of North America. Tree Physiol 7:227-238

Buchfink B, Xie C, Huson DH (2015) Fast and sensitive protein alignment using DIAMOND. Nat Methods 12(1):59-60

Grabherr MG, Haas BJ, Yassour M, Levin JZ, Thompson DA, Amit I, Adiconis X, Fan L, Raychowdhury R, Zeng Q, Chen Z, Mauceli E, Hacohen N, Gnirke A, Rhind N, di Palma F, Birren BW, Nusbaum C, Lindblad-Toh K, Friedman N, Regev A (2011) Full-length transcriptome assembly from RNA-seq data without a reference genome. Nat Biotechnol 29(7):644-652
Huang Q, Wang Y, Li B, Chang J, Chen M, Li K, Yang G, He G (2015) TaNAC29, a NAC transcription factor from wheat, enhances salt and drought tolerance in transgenic Arabidopsis. BMC Plant Biol 15(1):268

Kremer A, Abbott AG, Carlson JE, Manos PS, Plomion C, Sisco P, Staton ME, Ueno S, Vendramin GG (2012) Genomics of Fagaceae. Tree Genet Genomes 8(3):583-610

Livak KJ, Schmittgen TD (2001) Analysis of relative gene expression data using real-time quantitative PCR and the $2^{-\Delta \Delta \mathrm{Ct}}$ method. Methods 25(4):402-408

Martin M (2011) Cutadapt removes adapter sequences from highthroughput sequencing reads. Embnet J 17(1):10-12

Ren X, Chen Z, Liu Y, Zhang H, Zhang M, Liu Q, Hong X, Zhu JK, Gong Z (2010) ABO3, a WRKY transcription factor, mediates plant responses to abscisic acid and drought tolerance in Arabidopsis. Plant J 63(3):417-429

Sakuma Y, Maruyama K, Qin F, Osakabe Y, Shinozaki K, YamaguchiShinozaki K (2007) Dual function of an Arabidopsis transcription factor DREB2A in water-stress-responsive and heat-stress-responsive gene expression. Proc Natl Acad Sci 103(49):18822-18827

Tarkka MT, Herrmann S, Wubet T, Feldhahn L, Recht S, Kurth F, Mailänder S, Bönn M, Neef M, Angay O, Bacht M, Graf M, Maboreke H, Fleischmann F, Grams TEE, Ruess L, Schädler M, Brandl R, Scheu S, Schrey SD, Grosse I, Buscot F (2013) OakContigDF1591, a reference library for studying differential gene expression in Quercus robur during controlled biotic interactions: use for quantitative transcriptomic profiling of oak roots in ectomycorrhizal symbiosis. New Phytol 199(2):529-540

Torre S, Tattini M, Brunetti C, Fineschi S, Fini A, Ferrini F, Sebastiani F (2014) RNA-Seq analysis of Quercus pubescens leaves: De Novo transcriptome assembly, annotation and functional markers development. PLoS ONE 9(11):e112487

Ueno S, Provost GL, Léger V, Klopp C, Noirot C, Frigerio JM, Salin F, Salse J, Abrouk M, Murat F, Brendel O, Derory J, Abadie P, Léger P, Cabane C, Barré A, de Daruvar A, Couloux A, Wincker P, Reviron MP, Kremer A, Plomion C (2010) Bioinformatic analysis of ESTs collected by Sanger and pyrosequencing methods for a keystone forest tree species: oak. BMC Genom 11:650

Xiang Y, Tang N, Du H, Ye H, Xiong L (2008) Characterization of OsbZIP23 as a key player of the basic leucine zipper transcription factor family for conferring abscisic acid sensitivity and salinity and drought tolerance in rice. Plant Physiol 148(4):1938-1952

Yang W, Liu XD, Chi XJ, Wu CA, Li YZ, Song LL, Liu XM, Wang YF, Wang FW, Zhang C (2010) Dwarf apple MbDREB1 enhances 
plant tolerance to low temperature, drought, and salt stress via both ABA-dependent and ABA-independent pathways. Planta 233:219-229
Publisher's Note Springer Nature remains neutral with regard to jurisdictional claims in published maps and institutional affiliations. 